Procedure.-Removal of base of first phalanx and head of first metatarsal, right foot. The metatarsal head was found to be necrotic. Recovery was uneventful, and the patient was discharged a few weeks later. In December, 1949 , the adjacent second toe was amputated because of necrosis of the terminal phalanx.

Readmitted January, 1950, with redness and swelling of the second left toe. $X$-ray film showed "necrosis of the tip of the terminal phalanx." Local amputation of the affected digit was carried out under general anaesthesia. There has been no recurrepce, and the patient can walk about unaided (see Fig. 3).

\section{Case 4}

A woman aged 47. Diabetic for five years, taking 20 units protamine zinc insulin and 10 units soluble insulin daily. Admitted Februáry, 1950, with swelling and inflammation of the fourth right toe and lymphangitis of the dorsum of the foot. $X$-ray film showed "erosion of the middle phalanx." Systemic penicillin was administered and the inflammatory condition subsided. No surgical intervention was advised at that time.

Readmitted May, 1950, with recurrence of pain and swelling in the same toe.

Procedure.-Removal of the affected toe under general anaesthesia. Good functional result, and no evidence of recurrence since discharge (see Fig. 4).

The first three patients had all been advised elsewhere to have amputation performed, either of the foot only or at a higher level. All of them are still fully ambulant despite the loss of one or more toes.

\section{Discussion}

Are not these results in contrast with those obtained in non-diabetic senile gangrene, which almost inevitably necessitates above-knee amputation? It has been our experience that gangrene in the non-diabetic, even without thrombosis in the main vessels, has not been amenable to treatment by conservative surgery, which can give such encouraging results in the diabetic patient of comparable age. The question thus arises, Why should such a difference exist in patients of similar age and with a comparable degree of vascular degeneration where one is a diabetic and the other not?

The superimposed infection is currently believed to be the dominating factor in diabetic gangrene, and the good results of conservative surgery are attributed to the adequate control of the infection. It is our opinion, however, that the control of the infection is only a secondary factor, and we venture the suggestion that in the diabetis an adequate collateral circulation is already in existence.

It is known that diabetes mellitus predisposes to early degenerative changes in the intima of arteries, and various reasons for this have been suggested, such as increased blood cholesterol and high blood fat, but it is still open to question whether these theories provide the complete explanation. There is no doubt, however, that histological examination of the blood vessels of young diabetics often shows intimal proliferation with deposits of cholesterol crystals and lipid-containing cells. Joslin et al. (1946) quote Case 1305, a boy of 16, in whose aorta atheromatous plaques were found; similar findings in young adults are described by Smith (1925) and Oppenheimer and Fishberg (1925).

Joslin and his colleagues hold the view, and our own results support it, that the slowly progressive occlusion of the vascular lumen stimulates the development of collaterals in the limb. When, therefore, complete obstruction occurs in the smaller vessels, this collateral circulation maintains satisfactory nutrition in the neighbourhood. Control of infection in the affected toe either by simple débridement and drainage or by local amputation, will prevent spread to adjacent parts and thus hinder thrombosis in vessels which are still patent.

On the other hand, the non-diabetic with senile medial sclerosis, who suddenly develops a thrombus in one of the large vessels, has no compensatory mechanism, and so widespread necrosis takes place below the level of the obstruction.

\section{Summary}

Gangrene in the diabetic is extremely amenable to conservative surgery, and a radical amputation should never be performed as a routine measure. The theory is advanced that, although control of infection of the gangrenous tissue is of paramount importance, the diabetic state stimulates the formation of a collateral circulation which is usually well established when the crisis of peripheral vascular thrombosis supervenes.

We should like to express our thanks to Professor A. M. Boyd, Department of Surgery, Royal Infirmary, Manchester, for his helpful criticism and encouragement in the preparation of this paper.

\section{REFERENCES}

Joslin, E. P., Root, H. F., White, P., Marble, A., and Bailey, C. C. (1946). The Treatment of Diabetes Mellitus, 8 th ed. London.

Oppenheimer, B. S., and Fishberg, A. M. (1925). Arch. intern. Med., 36, 667 .

Smith, M. G. (1925). Bull. Johns Hopk. Hosp., 36, 203.

\section{ASSOCIATED PERFORATION AND HAEMORRHAGE IN PEPTIC - ULCERS TREATMENT BY IMMEDIATE GASTRECTOMY}

BY

\author{
N. S SLATER, M.B., F.R.C.S. \\ Surgical Chief Assistant, Edgware General Hospital
}

While perforation and haemorrhage are both common complications of peptic ulceration, they do not, fortunately, often occur simultaneously. Gordon-Taylor (1937) has classified these associated disasters into three groups: (1) haemorrhage and perforation occurring concomitantly, (2) haemorrhage following a recently treated perforation, and (3) perforation occurring after haemorrhage.

I wish to report three recent cases which fall into the first group and in which the two complications presented together. Case 3 is unusual in the pathological findings.

\section{Case 1}

A man aged 40 was admitted as an emergency on May 22 , 1950 , having had a sudden onset of generalized abdominal pain six hours previously. He had vomited several times in the interval and brought up "coffee grounds." A five-year history suggestive of peptic ulceration was obtained, and clinically he presented characteristic features of a perforated peptic ulcer. In addition he was very shocked and showed marked pallor. His blood pressure was $100 /$ ? $\mathrm{mm}$. $\mathrm{Hg}$, the radial pulse was of poor volume, and the peripheral circulation showed signs of collapse. A Ryle's tube obtained altered blood from the stomach. Pre-operatively 2 pints (1.14 litres) of blood was given followed by plasma, and two hours later his general condition had improved, his blood pressure rising to $120 / 90 \mathrm{~mm}$. $\mathrm{Hg}$.

At operation, under general anaesthesia, a perfcrated ulcer was found on the antero-superior aspect of the first part of the duodenum. The peritoneal cavity was full of 
blood and exudate, the former being seen to come from the perforation. A gastrectomy, removing the ulcer, was performed with an antecolic Polya gastro-jejunostomy. The removed stomach and duodenum showed an extensive duodenal ulcer, which had eroded into three large branches of the right gastric artery, their open mouths being visible in the floor of the ulcer.

The immediate post-operative convalescence was uneventful, but seven days after operation a remittent fever and physical signs at the right base suggested a developing subphrenic abscess. Screening of the chest showed no movement of the right cupola of the diaphragm. He was given penicillin systemically and 2 further pints (1.14 litres) of blood. Within a week there was a marked clinical improvement, and the pyrexia subsided. When the chest was screened again 10 days later diaphragmatic movement was found to be full. The patient was discharged symptomfree and eating well on June 15 . When subsequently seen in the "follow-up" clinic his progress was maintained; he had returned to a normal diet and had had no further indigestion.

\section{Case 2}

A man aged 52 was admitted as an emergency on October 10, 1950, having had a sudden onset of generalized abdominal pain four hours previously. He had vomited once and brought up "coffee grounds." A history of peptic ulceration covering 10 to 15 years was obtained, but there was no suggestion of previous haemorrhage. Clinically he presented the classical signs of a perforated peptic ulcer, and was not unduly shocked or pale. His blood pressure was $140 / 100 \mathrm{~mm}$. Hg. A Ryle's tube obtained altered blood in moderate quantity from the stomach.

At operation, under general anaesthesia, a perforated ulcer was found on the antero-superior aspect of the first part of the duodenum, through which poured altered and fresh blood. A high gastrectomy was performed with an antecolic valvular anastomosis. The excised stomach and duodenum showed a duodenal ulcer which had eroded a large branch of the right gastric artery. During and after operation he received 2 pints (1.14 litres) of blood. His post-operative convalescence was quite uneventful, and he was discharged on October 24. When seen in the "followup" clinic he looked extremely well and was on a normal diet and free of dyspepsia.

\section{Case 3}

A man aged 60 was admitted on October 29, 1950. He had a history of sudden severe abdominal pain which had come on eight hours previously. He had twice vomited fresh blood before admission and brought up a further 8-12 oz. (230-340 ml.) of bright blood in the receivingroom. There was a history of indigestion covering a period

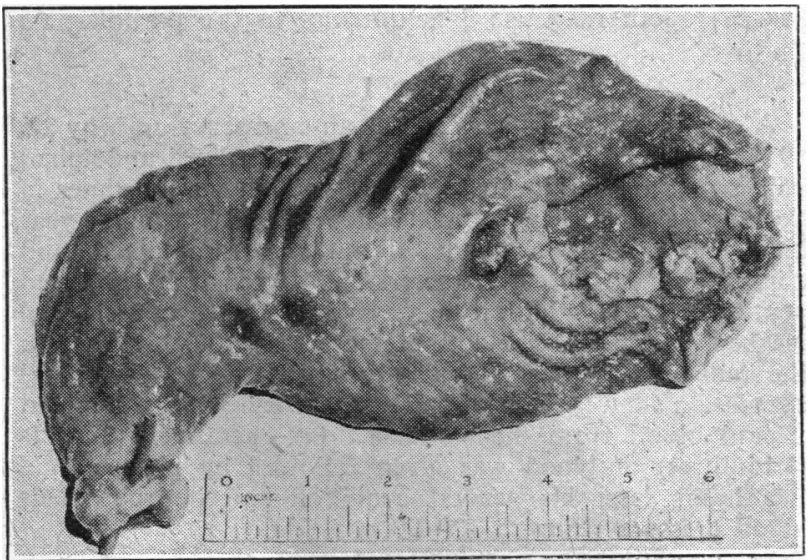

Photograph of stomach resected from Case 3. The highest of the three gastric ulcers was bleeding and the duodenal ulcer had perforated. of several years and a possible melaena stool 12 hours before admission. On examination his abdomen showed signs typical of a perforated peptic ulcer. In addition he was very pale and shocked, his blood pressure being $70 / 50 \mathrm{~mm}$. Hg. An immediate transfusion was begun, and within two hours his blood pressure had risen to $100 / 75 \mathrm{~mm}$. $\mathrm{Hg}$, with considerable clinical improvement. He was then considered fit for operation.

Laparotomy, under general anaesthesia, showed a perforated ulcer on the antero-superior aspect of the first part of the duodenum, from which poured considerable quantities of altered and fresh blood. - Routine palpation of the stomach revealed the presence of a large gastric ulcer high on the lesser curvature, firmly adherent to the pancreas. It appeared possible, therefore, that a gastric ulcer was responsible for the bleeding. A high gastrectomy was performed, with an antecolic Polya gastro-jejunostomy. Ligation of the left gastric vessels presented some difficulty, as they were involved in the ulcer bed. The specimen removed (see illustration) showed that there were, in fact, four ulcers: a large high lesser-curve ulcer eroding a branch of the left gastric artery, this being responsible for the haemorrhage; two smaller shallow ulcers on the lesser curvature; and a perforated duodenal ulcer.

The immediate post-operative convalescence was uneventful, but five days after operation the urinary output diminished in spite of adequate fluid intake and attention to electrolyte balance. The patient finally became anuric, dying on the ninth post-operative day with a blood urea of $375 \mathrm{mg}$. per $100 \mathrm{ml}$. Necropsy showed generalized oedema, marked in the lungs, and the kidneys had a mottled, pale, swollen cortex. On section these showed the histological appearances of a "lower nephron nephrosis." These changes could have been caused by a period of kidney anoxia due to the gross anaemia resulting from the recent severe blood loss, and by shock with a blood pressure little over $70 \mathrm{~mm}$. $\mathrm{Hg}$ for several hours before operation. The site of operation appeared satisfactory.

\section{Discussion}

There is an axiom which states, "Bleeding peptic ulcers do not perforate, and perforated ulcers do not bleed." Anatomical reasons for this in duodenal ulcers have been advanced; and an anaemic spot has been described on the anterior aspect of the first part of the duodenum, where most such ulcers occur (Einhorn, 1930). However, in most reviews of peptic ulceration complicated by perforation $20 \%$ of cases give a previous histcy of bleeding. Further, the double complication is said to occur in between 1 and $3 \%$ (Baker, 1947 : Gordon-Taylor, 1937) and $13 \%$ (Olson and Norgore, 1946). The lower figures would probably be more representative of experience in this country.

I have found no reference in recent American or British literature to concomitant bleeding in one peptic ulcer and perforation of another, though Kaye (1946) describes perforation of a pre-pyloric ulcer and six days later a fatal haemorrhage from a chronic high lessercurve gastric ulcer. There is a similarity between his case and Case 3, the difference in the timing of the two complications being probably fortuitous. In addition, the two uncomplicated ulcers of the lesser-curve make Case 3 an unusual one. It is interesting to note, in passing, that neither of these two gastric ulcers was palpable at operation, being discovered only on examination of the excised stomach. In operating for a suspected bleeding peptic ulcer, the absence of external indication or palpable evidence should not, therefore, too easily deter the surgeon (Ives, 1949), nor should it be too hastily assumed that an obvious ulcer is necessarily the one causing the haemorrhage. It is by no means uncommon, on examination of "cold" gastrectomy specimens, to find 
evidence of associated gastric and duodenal ulcers, or multiple lesions in the stomach or duodenum.

Gordon-Taylor (1937), in his classical review of the part surgery may play in the treatment of bleeding peptic ulcers, divides the problem of associated haemorrhage and perforation into the three groups mentioned above. He points out the great difference in prognosis between those cases in which the bleeding occurs at the same time as perforation (group 1) and of those in which either of the two complications occurs during recovery from the other (groups 2 and 3). He was able to quote five personal cases in the first group with no mortality. Four of these involved gastric ulcers and one a duodenal ulcer.

Where haemorrhage arises from an ulcer which has recently been sutured for perforation (group 2) all authorities agree that medical measures should be pursued, and, though such haemorrhage is a complication of the most calamitous nature, recovery does sometimes take place.

Where perforation has complicated a bleeding peptic ulcer (group 3) operation is indicated, though a large proportion of cases are not fit for any form of surgery. Gordon-Taylor (1937) states that he has never seen a recovery with or without operation. In a later paper (Gordon-Taylor, 1946) he quotes Avery Jones, who regards severe pain before, and particularly when it persists after, haemorrhage as a point of bad prognostic significance and a possible indication for surgical intervention. The diagnosis of perforation may be difficult in a shocked exsanguinated patient. If an early perforation is not to be missed, surgical treatment, where the patient can be made fit for it, would therefore seem urgently indicated when ulcer pain persists after haemorrhage.

\section{Summary}

The subject of associated haematemesis and perforation in peptic ulcers is reviewed. Three cases are presented in which the double complication occurred simultaneously.

Treatment by immediate gastrectomy is discussed.

I should like to thank Mr. Frank Forty, under whom these cases were admitted, for allowing me to operate on them and for the encouragement and stimulating advice he has given both in the management of the cases and in making this record. I am grateful to Miss N. H. Shaw, who is responsible for the photography.

REFERENCES
Baker, Charles (1947). Guy's Hosp. Rep., 96, 1.
Einhorr, M. (1930). Surr. Gynec. Obstet,.,50, 416.
Gordon-Taylor, G. (1937). Brit. J. Surg., 25, 403.
Ives, (1946). Ibid., 33, 336.
Kaye, M. (1949). Lancet, 2, 644.
Olson, H. B., and Nritish Medical Journal, 2, 695.

At a joint meeting of psychiatrists and chest physicians held by the Royal Medico-Psychological Association and N.A.P.T. on November 15 Dr. Andrew Morland discussed the reasons why the incidence of tuberculosis in mental hospitals was seven or eight times that in the general population. He said that although the chances of infection were greater, he felt that the lowered resistance of mental patients due to fears and depressions was a more likely cause. Dr. R. G. Maclaren thought that with mental patients the three chief evils were overcrowding, particularly at night, the grave shortage of nurses, and the low'standard of dietary. Also patients sometimes refused to eat and needed much individual attention to get them to do so. Dr. B. T. Mann pointed out the risk to the community at large owing to the high proportion of mental patients discharged each year. Dr. Freida R. Hendeles described the organization of a tuberculosis unit in a mental hospital, and advocated a bed provision of $2 \frac{1}{2} \%$ of the total beds to be served.

\section{BRONCHIECTASIS WITH UNSUSPECTED FOREIGN BODY}

BY

\author{
B. P. MOORE, M.B., B.S., B.Sc., F.R.C.S. \\ Surgical First Assistant, London Chest Hospital
}

Four cases of bronchiectasis admitted to one chest hospital during the last six years, and treated by resection, were found to have an entirely unsuspected intrabronchial foreign body in the portion of lung removed. In none of them was there any history to suggest the inhalation of a foreign body, even on direct questioning after operation.

\section{Case 1}

An unmarried woman aged 22 , of no occupation, was admitted on January 15,1948 . She had had pneumonia with haemoptysis in 1945. A further haemoptysis occurred in 1946 (a cupful), and another in 1947, when the severity demanded a blood transfusion. Between these attacks and her admission she had no complaints.

On examination the patient appeared to be a normal healthy young woman. Her weight was 9 st. $2 \mathrm{lb}$. (58 kg.), temperature $98.4^{\circ}$ F. $\left(36.9^{\circ}\right.$ C. $)$, pulse 90 , and blood pressure $114 / 80$. Air entry was impaired in the left upper zone of the chest. Repeated sputum examinations revealed a mucopurulent trace, but no tubercle bacilli. A blood count showed: red cells, 4,000,000; haemoglobin, $80 \%$; white cells, 9,000. A chest radiograph showed a slight increase in striation in the left lower zone. Bronchoscopy revealed only mucopurulent secretion from the left upper lobe orifice. Bronchography showed lingular and left anterior bronchiectasis; the remaining branches were normal.

Operative treatment was indicated by repeated haemorrhage, and on March 4 resection of the affected lingular and left anterior segments was successfully performed. The post-operative course was satisfactory, and she was discharged on March 13. A follow-up report by her doctor stated that she was well and free from respiratory symptoms.

Examination of the specimen showed bronchiectasis with infection and fibrosis affecting the lingular and left anterior bronchi. Within the bronchus was a mass of inspissated material containing vegetable seed cases and stalks. Microscopical sèction showed considerable submucosal infection with spherical hyaline bodies, probably also of vegetable origin.

No history was obtained before or after operation of an incident in which the grass seeds might have been inhaled, but on direct questioning after operation the girl's mother said that as a child the patient would often chew stalks of grass.

\section{Case 2}

An unmarried woman, a houseworker aged 32, was admitted on December 30, 1947. In May, 1947, dental extractions were carried out under general anaesthesia. A few weeks later she had a cough with foul sputum, occasionally streaked with blood, and in October she was treated for lung abscess with penicillin and postural drainage. Up to the time of admission she still had a cough with a little purulent sputum.

On examination she appeared to be a healthy woman. Her weight was 6 st. 2 lb. (39 kg.), temperature $98.4^{\circ} \mathrm{F}$. $\left(36.9^{\circ}\right.$ C. $)$, and pulse 80 . Her sputum was fetid, purulent, up to $\frac{1}{2}$ oz. (14 ml.) in quantity, and contained no tubercle bacilli on repeated examination. Chest examination revealed impaired movement and air entry with occasional added moist sounds in the right upper zone anteriorly. Her blood pressure was $120 / 70$, haemoglobin $100 \%$, white cells 16,000 . A chest radiograph revealed a streaky opacity in the right anterior region, and a dilated bronchus could be seen in the 